\title{
Guidelines for the management of asthma in adults
}

\author{
An excellent first effort
}

Earlier this year the British Thoracic Society, combined with the Royal College of Physicians, the King's Fund, and the National Asthma Campaign, brought together 34 participants, received 357 answered questionnaires, and took the views of an open debate at the society's meeting before producing guidelines on the management of asthma. Despite this parentage - for multiple authors often seem to produce an excess of muddled words - the guidelines are clear, relevant, and practical (though not brief). They deserve careful consideraton from all those who care for patients with asthma. The first part, the management of chronic asthma, appeared last week (p 651). ${ }^{1}$ The second part, on acute severe asthma, is published this week on $\mathrm{p} 797 .^{2}$

These statements should be seen as guidelines and not as didactic rules for treatment. The danger is that "recipe book" treatment may limit the use of common sense and clinical judgment, but against that must be set the rising morbidity and mortality from asthma in Britain and around the world, which amply justify this initiative. Individual practice will vary, but well over $90 \%$ of members who replied to the British Thoracic Society questionnaire thought that the guidelines were appropriate for hospital staff and general practitioners, though only $80 \%$ thought that the practice described was achievable. Given this consensus nothing revolutionary would be expected in the guidelines, most of the information being widely available in current publications on asthma. Nevertheless, the stamp of authority from the British Thoracic Society and the other bodies may lead to wide acceptance and use. These guidelines should be read by all health staff who manage patients with asthma - the flow chart for acute asthma provides a simple, practical scheme for accident and emergency departments. The guidelines will also form an excellent basis for clinical audit; they can be used to assess local performance and to discuss the ability and desirability of treating patients according to the recommendations.

A few recommendations may cause minor controversy. Those on the use of intravenous aminophylline carry some ambiguity, and the relative importance of ipratropium bromide in acute asthma and of sodium cromoglycate and nedocromil sodium in chronic asthma will cause some disagreement. These are topics for debate and further investigation.

Though the guidelines should meet with general approval in Britain, they would be more revolutionary elsewhere. For example, doctors in North America still use theophyllines too freely but give inhaled corticosteroids in doses British doctors think too low. ${ }^{3}$ Many international allergists may be surprised (and worried about their income) on reading the statement that hyposensitisation has no place in treating asthma.

Recent research has highlighted the relevance of the inflammatory component in the airways, and the guidelines emphasise the importance of early anti-inflammatory treatment. Inhaled corticosteroids are the main element of this treatment; sodium cromoglycate and nedocromil sodium are given less emphasis. The threshold suggested for such treatment is the patient using an inhaled bronchodilator more than once daily or having symptoms at night. These recommendations provoked debate at the thoracic society meeting. This approach is known to reduce symptoms and short to medium term morbidity, but we can only hope that it is also beneficial in the long term. The criteria should prompt relevant questions about nocturnal symptoms. ${ }^{+}$

Naturally enough, the guidelines start with patients in whom asthma has been diagnosed. In patients with chronic airflow obstruction and limited reversibility the management plans begin to creak a little, particularly for long term care.

Asthma is common and most of the care must, and should, be provided by general practitioners. The guidelines give suggested indications for referring patients to hospital specialists. Both in hospital and general practice asthmatic patients do better when seen by someone with an interest in the condition. This is shown, for instance, by better control and fewer readmissions in patients admitted with their acute attack into wards with a special interest in respiratory medicine rather than general wards. ${ }^{5}$ The importance of the patient's own participation in management is also emphasised, and this will be much easier with the availability of peak flow meters on prescription and the development of asthma clinics in general practice.

The useful life span of consensus guidelines is necessarily limited, and before long the participants will need to start again. Such a policy is to be welcomed as both the treatment and understanding of asthma change with time. The main management change in the near future is likely to come with the advent of long acting inhaled $\beta$ agonists that will need to be administered only every 12 hours. ${ }^{6}$ Unless it is carefully handled this simple and effective regimen may tend to reduce the use of anti-inflammatory treatment in favour of sustained bronchodilatation alone. Such treatment may give general 
good control but leave open the risk of acute, severe exacerbations.

P JOHN REES

Consultant Physician,

United Medical and Dental Schools,

Guy's Hospital,

London SE1 9RT

1 Brewis G. Guidelines for the management of asthma in adults. I. Chronic asthma. Br Med $\mathcal{F}$ 1990;301:651-3.

Brewis G. Guidelines for the management of asthma in adults. II. Acute severe asthma. Br Med $\mathcal{J}$ 1990;301:797-800

Lam A, Newhouse MT. Management of asthma and chronic airflow limitation. Are methylxanthines obsolete? Chest 1990;98:44-52.

Turner-Warwick M. On observing patterns of airflow obstruction in chronic asthma. Br f Dis Chest 1977;71:73-86.

Bucknall CE, Robertson C, Moran F, Stevenson RD. Differences in hospital asthma management. Lancet 1988;i:748-50.

Ullman A, Svedmyr N. Salmeterol, a new long acting inhaled beta 2 agonist: comparison with salbutamol in adult asthmatic patients. Thorax 1988;43:674-8.

\section{Oxalate, livers, and kidneys}

\section{Combined renal and hepatic transplants transform the outlook in primary hyperoxaluria type 1}

The incidence of calcium oxalate nephrolithiasis is reaching epidemic proportions in Western societies, in which it is usually associated with idiopathic hypercalciuria. Many of the patients who have recurrent calcium oxalate stones have hyperoxaluria, which, although mild, assumes greater importance because of its disproportionate effect on the solubility product for calcium oxalate. ${ }^{12} \mathrm{~A}$ few patients, however, present with particularly aggressive recurrent oxalate urolithiasis caused by excessive oxalate biosynthesis due to deficiency of hepatic peroxisomal alanine:glyoxylate aminotransferase (primary hyperoxaluria type 1). These patients usually present in childhood and are dead by the age of 30 . Until recently their prognosis was hopeless, but it has been transformed by aggressive dialysis with early renal transplantation, and, in particular, orthoptic liver transplantation.

Primary hyperoxaluria type 1 needs to be distinguished from type 2, a deficiency of $\mathrm{D}$-glycerate dehydrogenase (glyoxylate reductase) which is not confined to the liver, ${ }^{34}$ and from type 3 , due to oxalate hyperabsorption. ${ }^{5}$ The enzyme defect may be diagnosed biochemically in a specimen taken by percutaneous needle liver biopsy. ${ }^{6}$ Occasionally patients present with end stage renal disease at any age from the neonatal period onward, and diagnostic liver biopsy is of particular help in these cases.

Pyridoxal phosphate is the cofactor for alanine:glyoxylate aminotransferase, and in some patients with primary hyperoxaluria type 1 pharmacological doses of pyridoxine reduce urinary oxalate concentrations. But for those not responding to pyridoxine the outlook has been bleak. Until recently the results of renal transplantation have been poor because the graft has been destroyed through rapid oxalate deposition in the perioperative period and the later development of obstruction due to calculi in the collecting system. Treatment by conventional dialysis regimens only prolongs life, and instead of dying quickly from uraemia patients die miserably after possibly years of increasing morbidity because of the serious effects of extrarenal systemic deposition of oxalate (oxalosis). These complications include painful osteodystrophy, cardiomyopathy and cardiac conduction defects, peripheral neuropathies and mononeuritis multiplex, synovitis, arteriopathy with disseminated occlusive vascular lesions, and ulcerating subcutaneous calcium oxalate calcinosis.

Once the creatinine clearance falls below about $25 \mathrm{ml} / \mathrm{min} /$ $1.73 \mathrm{~m}^{2}$ the combination of continued oxalate overproduction by the liver and reduced oxalate excretion in the urine produces a rapid increase in body oxalate concentrations and the development of systemic oxalosis. ${ }^{7}$ Any acute reduction in renal function, produced, for example, by ureteric obstruction, may lead to extensive renal oxalosis and irreversible acute on chronic renal failure; patients who reach this stage when no plans have been made for their future management have a very poor prognosis.

It is now clear that the chances of successful renal transplantation are improved if it is performed while there is some useful residual renal function and before serious systemic oxalosis has developed. ${ }^{8}$ Vigorous perioperative haemodialysis to keep plasma oxalate concentrations as low as possible and maintaining high urine volumes throughout reduces the risk of stone formation. Two of our patients treated in this way have normal graft function four years and three and a half years after transplantation, and one has completed a successful pregnancy. ${ }^{9}$ The risk of stone formation in the transplant can be reduced by diuresis and crystallisation inhibitors such as magnesium or phosphate ions, and any stones that do form can be dealt with by percutaneous nephrolithotomy or extracorporeal shock wave lithotripsy.

The definitive treatment of primary hyperoxaluria type 1 by simultaneous hepatic and renal transplantation was first successfully accomplished about four years ago; this patient remains in full time employment, with near normal renal function..$^{10}$ An earlier patient in whom biochemical correction was proved died from an opportunistic infection eight weeks after the operation. ${ }^{11}$ About 30 further cases world wide have now been treated in this way, with gratifying results. The combined operation corrects the underlying defect in the liver and replaces the damaged end organ, the kidney, which is then no longer subject to attack by oxalate overproduction.

Heterotopic auxiliary liver transplantation has been proposed because it does not necessitate removing the whole of the patient's liver. Nevertheless, glyoxylate accumulating behind the metabolic block in the patient's liver peroxisomes is unlikely to reach the normal alanine:glyoxylate aminotransferase in the donor liver peroxisomes because lactate dehydrogenase, which catalyses the oxidation of glyoxylate to oxalate, is highly active in liver cytosol and blood plasma. Furthermore, competition from the patient's own liver, which functions normally except for the alanine:glyoxylate aminotransferase deficiency, would also militate against a successful liver graft in these patients.

These recent developments have raised several questions. Should these patients be offered an orthotopic liver transplant before renal damage has occurred? ${ }^{12}$ Alternatively, should patients entering end stage renal failure be treated firstly by a kidney transplant alone, with aggressive perioperative dialysis and a well matched kidney from a living related donor, with hepatic transplantation being held in reserve? Finally, should the first step for a patient established on dialysis with the complications of oxalosis be a liver transplantation to cut off excessive oxalate synthesis followed by a kidney transplant later, when the miscible oxalate pool has been depleted by vigorous haemodialysis or haemofiltration? The reverse of this strategy, with renal transplantation 250 days before liver transplantation, has also been reported. ${ }^{13}$ The relative merits of these strategies are still being evaluated. Clearly, however, the dramatic and curative advance was the application of orthotopic liver transplantation. These patients demand a high level of collaboration between several disci- 